\title{
EXPERIMENTAL INVESTIGATION OF SECONDARY FLOWS IN A LOW HUB-TIP RATIO FAN
}

\author{
F. Nurzia and P. Puddu \\ Department of Mechanical Engineering \\ University of Cagliari \\ Cagliari, Italy
}

\begin{abstract}
The paper deals with an experimental investigation of the three-dimensional flow field at the exit of a low hub/tip ratio industrial axial fan. The relative flow was evaluated with fixed hot wire probes using the multisampling and phase-locked ensemble averaging techniques. Both mean flows and secondary flows are examined for different blade loading and stagger angles. The mean flows observed indicate a large separated flow region at the hub that is accentuated at the higher stagger angle. The secondary flows are particularly strong and affect the entire flow field. Their structure and location are also modified when stagger angle and blade loading are changed. The most complex situation is observed at the huh where a change in blade loading and stagger angle results in the interaction of the trailing vortex with the separated flow region, the passage vortex and midpassage region flow.
\end{abstract}

$\begin{array}{ll}\text { UST OF SYMBOLS } \\ \text { C } & \begin{array}{l}\text { absolute velocity } \\ \text { h }\end{array} \\ \text { ho } & \text { bladial distance from the hub blade } \\ \text { p.s. } & \text { pressure side } \\ \text { f } & \text { radius } \\ \text { 5.s. } & \text { suction side } \\ \text { s } & \text { circumferential coordinate } \\ \text { t } & \text { circumferential pitch } \\ U & \text { peripheral velocity at the rotor tip } \\ V & \text { hot wire cooling velocity } \\ W & \text { relative velocity } \\ x_{t}, x_{2}, x_{3} & \text { fixed cartesian coordinate system } \\ \Delta \mathrm{P}_{t} & \text { total pressure rise } \\ \beta & \text { relative flow yaw angle }\end{array}$

$\Psi=\frac{\Delta p_{l}}{\rho U^{2} / 2}$

$\Phi=\frac{C_{z}}{U}$

$\rho$

$\boldsymbol{\Omega}$

$\omega$

$\xi_{D}$

Subscript
$\mathbf{z , r}, \theta$
$n, t, b$
$s, \tau, n$
ref

Superscript

一 stagger angle from the blade chord to the axial direction

pitch angle

yaw angle

total pressure rise coefficient

flow rate coefficient

air density

angular rotor speed

vorticity

kinetic energy defect

axial, radial and tangential directions normal, tangential and binormal directions streamwise, transverse and normal directions reference flow 
updated; simplifications in the design, often introduced $10 \mathrm{cut}$ manufacturing costs, result in performances that in many cases are unacceptable nowadays;

- noise production depends, to some extent, on the aerodynamic design of the blades;

- the flow generated in turbomachines of this type is strongly three-dimensional and consequently such machines can be regarded as a useful test bench for studying three-dimensional viscous and non-viscous flow phenomena. At the same time they are a useful tool for improving three-dimensional computer codes for flow analysis using the Navier-Stokes equations.

The latter features lie in well with the numerous theoretical and experimental investigations on three-dimensional and viscous flow analysis in axial compressors (Dring et al., 1982; Adkins and Smith, 1982; Inoue and Kuroumaru, 1984; Gallimore and Cumpsty, 1986; Wisler et al., 1987; Dawes; 1987; Leylek et al., 1991; Goto, 1992) and in particular with those specifically concerned with the analysis of wall effects and of the vortices created and their streamwise evolution (Hunter and Cumpsty, 1982; Lakshminarayana el al., 1982; Pandya and Lakshminarayana, 1983; $\mathrm{Ha}, 1986$ : Inoue et al., 1986; Inoue and Kuroumaru, 1989; Chen et al., 1991; Stauter, 1992).

Performances can be only improved and the operational range of axial flow fans extended by gaining a betler underslanding of flow phenomena, as has happened for axial flow compressors.

The high stagger angles and low solidity, typical of axial flow fans, fall outside the range usually covered by experimental literature data making it impossible to use the well known correlation for the design and analysis problem.

The present investigation concerns the flow analysis of a widely used industrial axial fan whose relative flow pattem had already been studied to evaluate its general characteristics in design conditions (Puddu, 1992). More specifically here an experimental analysis has been conducted to study the secondary flow pattems and how they are affected by changes, in blade loading and stagger angle setting.

Relative flow was determined by measuring absolute velocity wilh a hot wire anemomeler, following the widely used periodic multisampling and phase locked ensemble averaging lechnique. In the case at hand a slanted single hot wire was placed at different radial slations and rotated around its axis 10 vary the relative position with respect to the flow. For the different operating conditions the distribution of mean velocity components is evaluated and the secondary flow patterns are represented. Analysis of the secondary flows shows the influence of the different vorlices as well as their strong interaction with the separated flow region at the hub.

\begin{tabular}{|c|c|c|c|c|}
\hline section & radius & chond & camber & thichess/chord \\
\hline $1-1$ & $159.39 \mathrm{~mm}$ & $116.34 \mathrm{~mm}$ & $0^{\circ}$ & 0.284 \\
\hline $2-2$ & $200.66 \mathrm{~mm}$ & $161.79 \mathrm{~mm}$ & $24^{\circ}$ & 0.151 \\
\hline 3-3 & $241.30 \mathrm{~mm}$ & $168.91 \mathrm{~mm}$ & $24^{\circ}$ & 0.110 \\
\hline 44 & $474.98 \mathrm{~mm}$ & $130.15 \mathrm{~mm}$ & $21^{\circ}$ & 0.073 \\
\hline
\end{tabular}
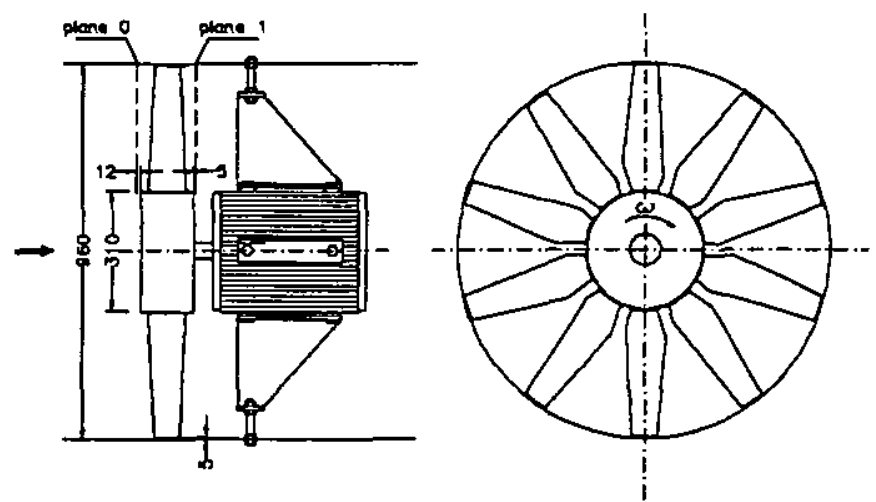

FIG.1 SCHEMATIC VIEW OF THE AXIAL FAN AND POSITION OF THE MEASURING PLANES

\section{TABLE 2. BLADE PROFILE COORDINATES}

\begin{tabular}{|c|c|c|c|c|c|c|c|c|}
\hline $\begin{array}{l}1-1 \\
x \mathrm{~mm}\end{array}$ & $\begin{array}{l}\text { S.S. } \\
\text { y } \mathrm{mm}\end{array}$ & $\underset{y \mathbf{m m}}{\text { p.S. }}$ & $\begin{array}{r}2-2 \\
x \text { m }\end{array}$ & $\begin{array}{c}\text { S.S. } \\
y \mathrm{~mm}\end{array}$ & $\underset{y \mathrm{~mm}}{\text { p.S. }}$ & $\begin{array}{r}3-3 \\
\times \mathrm{mmm}\end{array}$ & $\begin{array}{r}\text { S.S. } \\
\text { y mm }\end{array}$ & $\underset{y \mathrm{~mm}}{\text { p.s }}$ \\
\hline 58.17 & 0.00 & 0.00 & -79.07 & 0.00 & 0.00 & -81.56 & 0.00 & 0.00 \\
\hline-55.26 & 3.15 & -1.38 & -75.10 & 2.19 & -0.56 & .77 .60 & 2.49 & -0.03 \\
\hline .52 .35 & 4.16 & .269 & $\cdot 71.15$ & 3.13 & .108 & .73 .41 & 3.38 & -0.06 \\
\hline-46.53 & 6.11 & -5.18 & -63.25 & 4.97 & .203 & .65 .25 & 5.03 & -0.11 \\
\hline $.34,90$ & 9.59 & .9 .40 & -47.43 & 8.46 & -3.43 & -48.94 & 8.10 & -0.23 \\
\hline-23.27 & 1252 & .1254 & -31.62 & 11.57 & -4.35 & .3262 & 10.97 & -0.50 \\
\hline-11.63 & 14.74 & -14.73 & -15.81 & 14.28 & -4.99 & -16.31 & 13.34 & -0.80 \\
\hline 0.00 & 16.19 & .16 .16 & 0.00 & 16.25 & .5 .61 & 0.00 & 15.08 & -1.20 \\
\hline 11.63 & 16.50 & -16.50 & 15.81 & 17.46 & -6.22 & 16.31 & 16.20 & -1.62 \\
\hline 23.27 & 15.77 & -15.75 & 31.62 & 18.04 & -6.40 & 32.62 & 16.58 & -202 \\
\hline 34.90 & 14.14 & -14.20 & 47.43 & 16.92 & -6.23 & 8.94 & 15.50 & .2 .55 \\
\hline 46.53 & 10.95 & -10.95 & 63.25 & 1268 & $\cdot 5.51$ & 65.25 & 11.56 & $\cdot 3.11$ \\
\hline 52.35 & 8.19 & -8.18 & 71.15 & 8.42 & -4.80 & 73.21 & 7.68 & .3 .45 \\
\hline 55.26 & 5.79 & .5 .81 & 75.10 & 5.40 & -4.06 & 77.48 & 4.78 & -3.24 \\
\hline 56.71 & 3.96 & -4.05 & 77.08 & 3.25 & -3.31 & 79.52 & 2.94 & -274 \\
\hline 57.28 & 3.05 & -3.05 & 78.42 & 1.58 & -218 & 0.94 & 1.40 & -1.87 \\
\hline 58.17 & 0.00 & 0.00 & 79.06 & 0.00 & 0.00 & & 0.00 & 0.00 \\
\hline 4-4 & S.S. & p.s. & & & & & & \\
\hline $\mathrm{mm}$ & $y \mathrm{~mm}$ & $y \mathrm{~mm}$ & & & & & & \\
\hline-63.47 & 0.00 & 0.00 & & & & & & \\
\hline-60.35 & 1.59 & -0.49 & & & & & & \\
\hline .57 .24 & 1.65 & -0.78 & & & & & & \\
\hline 51.02 & 1.74 & -1.35 & & & & & & \\
\hline .38 .58 & 1.75 & .252 & & & & & & \\
\hline-26.13 & 1.74 & -3.93 & & & & & & \\
\hline-13.69 & 1.44 & .5 .55 & & & & & & \\
\hline-1.24 & 0.79 & -7.25 & & & & & & \\
\hline 11.20 & 0.25 & -9.10 & & & & & 2 & \\
\hline $\begin{array}{l}23.65 \\
36.09\end{array}$ & $\begin{array}{r}-1.83 \\
-435\end{array}$ & $\begin{array}{l}-11.15 \\
.13 .57\end{array}$ & & & & & & \\
\hline 48.54 & -8.84 & -16.69 & & & & & & \\
\hline 54.76 & -125 & -18.65 & & & & & & \\
\hline 57.87 & -15.0 & -19.83 & & & & & & \\
\hline 59.70 & -17.1 & -20.51 & & & & & & \\
\hline $\begin{array}{l}60.76 \\
60.98\end{array}$ & $\begin{array}{l}-18.5 \\
-19.55\end{array}$ & $\begin{array}{l}-19.97 \\
-19.55\end{array}$ & & & & & & \\
\hline
\end{tabular}

\section{EXPERIMENTAL FACILITY}

The industrial axial fan shown in Fig. 1 was used for the experiments. The experimental set-up complies with the slandard airway lype $C$ of the British Slandards 848 . The rotor, powered by a $17 \mathrm{~kW}$ eleciric motor at $1475 \mathrm{rpm}$, consists of len aluminium alloy blades with tip diameter of $950 \mathrm{~mm}$ (1ip clearance: $5 \mathrm{~mm}$ ) and hub diameter of $310 \mathrm{~mm}$. Some blade geometry characteristics for four blade sections are given in Table 1. For completeness in Table 2 the blade coordinates a1 these sections are also reported. The blade profiles which are 
modified Gottingen $\mathbf{4 3 6}$ for a large part of the blade span, are thicker and almost symmetric at the root where the flow suffers from high incidence conditions. The rotor blades have adjustable stagger angle and in these experiments two values $\left(\gamma=62^{\circ}\right.$ and $\left.y=70^{\circ}\right)$, estimated at the tip between the chord and the axial direction, were considered.

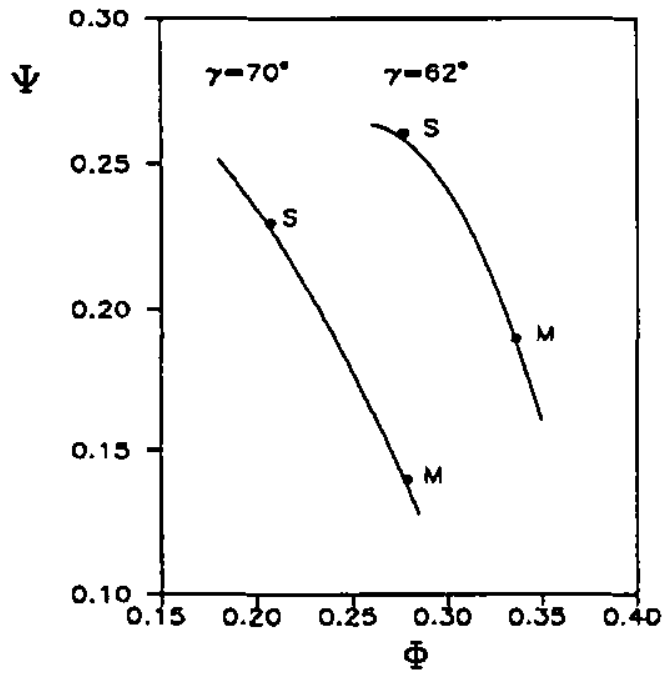

FIG.2 CHARACTERISTIC CURVES AND OPERATING CONDITIONSAT DIFFERENT STAGGER ANGLES.

\section{INSTRUMENTATION AND MEASURING TECHNIOUE}

The measurements of the relative flow field behind the rotor were made by a constant-temperature hot wire anemometer and a periodic multisampling and averaging lechnique with a computerized data acquisition system.

Two operating conditions corresponding to (M), high flow rate and (S) low flow rate (Fig.2), for the selected stagger angles $\gamma=62^{\circ}$ and $\gamma=70^{\circ}$ were considered. The sensors used were a $45^{\circ}$ slanted single hot wire of $5 \mu \mathrm{m}$ diameter (Dantec 55-P02) to measure instantaneous velocity and an aerodynamic five hole probe to measure mean flow characteristics.

The probes inserted from the casing were moved radially through different positions in the measuring plane shown in Fig.1. At each radial position 12 hot-wire probe orientations were set by rotating the probe axis and for each position 300 circumferential signals along two blade pitches were acquired every rotor revolution for 500 revs. The instantaneous signals were acquired at the sampling rate of $37 \mathrm{kHz}$ by means of a 12 hit AVD converter board and a magnetic pick-up which provides the pulse which triggers data acquisition. A low-pass filter and an amplifier were also included in the measuring system before acquisition to eliminate spurious signals and to improve measurement accuracy. The instantaneous cooling velocities, which are obtained from the instantaneous signals by analytical inversion of the King's law, are summed by the on-line computer during the 500 revolutions of the rotor 10 obtain the ensemble average of the cooling velocity.

In order to correctly determine the three components of the mean local velocity it is very important to know the angular response of the hot wire probe.

As reported by Puddu (1993), to characterise angular behaviour, the response of the hot wire was experimentally evaluated changing its orientation with respect to a known flow. The calibration curves thus obtained were approximated by the expression

$$
\mathrm{V}^{2}=\mathrm{K}_{\mathrm{d}} \mathrm{C}_{\mathrm{D}}^{2}+\mathrm{K}_{\mathrm{t}} \mathrm{C}_{\mathrm{t}}^{2}+\mathrm{K}_{\mathrm{b}} \mathrm{C}_{\mathrm{b}}^{2}
$$

where $K_{b}, K_{n}, K_{l}$ are the directional sensitivity coefficients, that are calibrated as a function of pitch and yaw angles and not regarded as constants, and $C_{b}, C_{b}, C_{l}$ are components of the velocity vector with respect to the intrinsic probe coordinate ( $\mathrm{n}$ t-b). The sensitivity coefficients are expressed analytically using second degree polynomials of the type:

$$
K_{b, n, t}=a_{0}+\sum_{i=1}^{2} a_{i} \varphi^{i}+b_{i} \psi^{i}
$$

whereas other workers (Lofdahl, 1986; Perdichizzi et al., 1990) assume them to be constant. These coefficients are determined using the least square method, comparing experimental data with the values calculated using Eq. (2). Following the same procedure described by Puddu (1993), we get the expression for determining mean flow that relates non linearly the mean flow components $\overline{C_{i}}$ to the measured mean cooling velocities $\bar{v}$.

$$
\bar{V}=\sum_{i=1}^{3} \sum_{j=i}^{3} A_{i, j} \overline{C_{i}} \overline{C_{j}}
$$

where the coefficients $A_{j, j}$ are a function of the directional sensitivity coefficients and of the probe orientations.

The mean velocity components can be calculated, from the 12 mean cooling velocities, by solving the system of non-linear equations (3).

The iterative procedure, used to solve this system, also takes into account, for each iteration, the variations of the sensitivity coefficients with probe orientation.

Using this technique, accurate mean velocity components can be obtained for a duct flow in the calibration wind tunnel (Puddu, 1993). Here in the mean flow field measurements the following uncertainties were estimated:

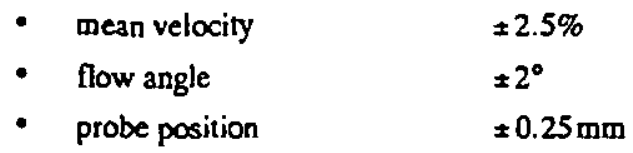

\section{EXPERIMENTAL RESULTS AND DISCUSSION}

The flow pattem up and downstream of the rotor has been determined along the blade span using aerodynamic probes as well as hot wire probes. 

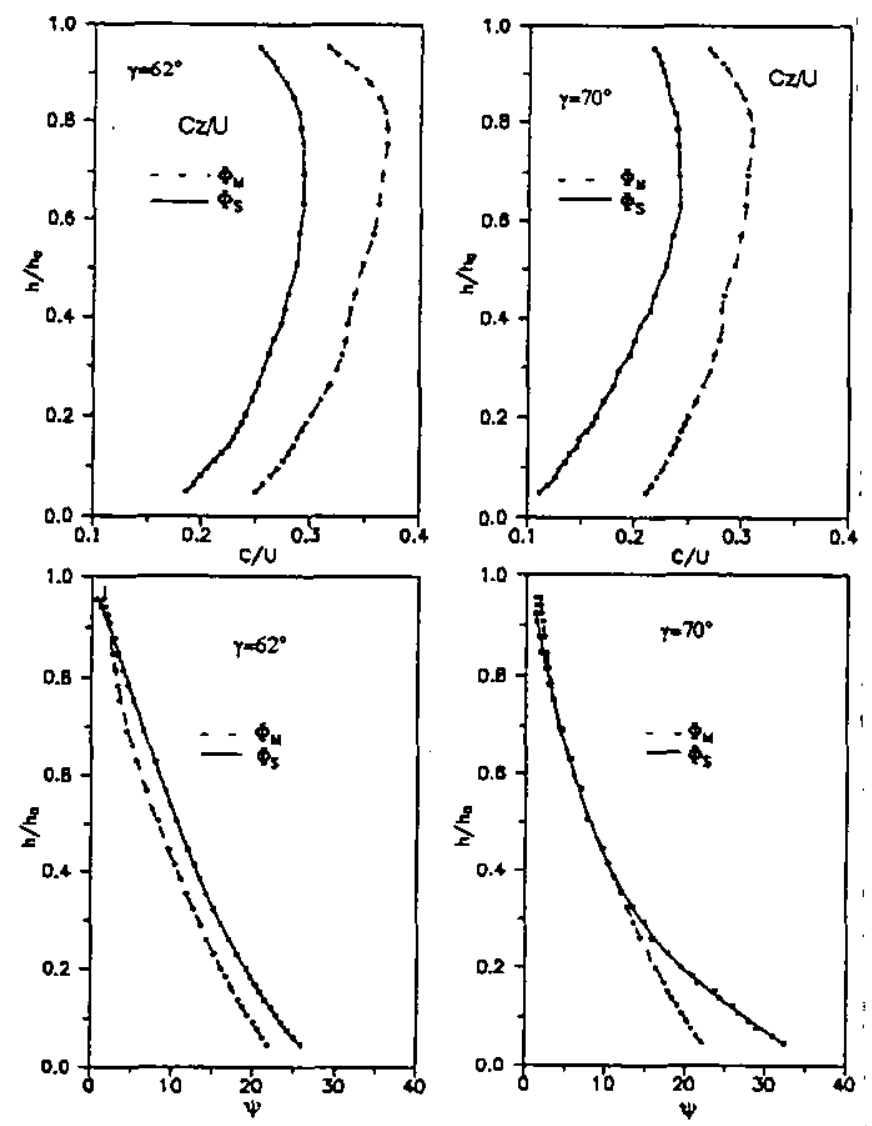

\section{FIG.3 MEAN AXIAL VELOCITY AND PITCH ANGLE AT THE ROTOR INLET}

Figure 3 shows the axial velocity component and the pitch angle distribution at the rotor inlet (measured with five hole probes). As can be seen, significant pitch flow angle variation exists in the hub region, aided also by hub geometry, which gives rise to strongly radial inlet flow. The axial velocity. distribution exhibits large variation associated to the curvature of the streamline near the hub region and to the development of the wall boundary layer along the duct at the tip.

The mean exit flow has been obtained by pitchwise averaging the hot wire measurements. Velocity distributions along the radius for different loads and stagger angles are plotted in Fig.4. The three velocity components are characterized by a constant gradient in the primary flow region but at the casing and at the hub stronger gradients appear. The hub is characterized by an extended separated flow region that broadens as the load and the stagger angle increase.

The contour plots of radial tangential and axial velocity components are presented in Fig.5 for the smaller stagger angle and highest flow rate $\Phi_{M}$.

Axial velocity gradients are evident in three regions: near the hub, where an extensive separated flow region exists, in the wakes and at the casing region both in the wall boundary layer
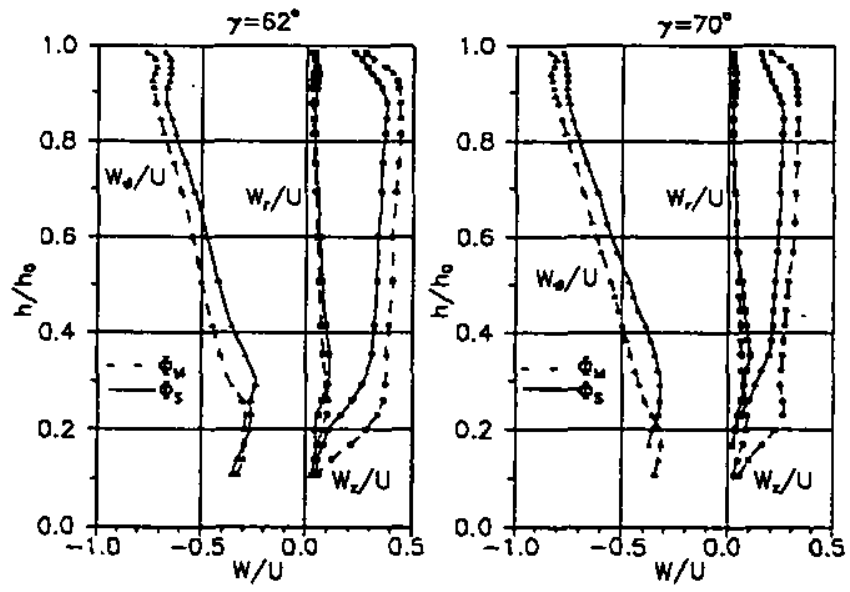

\section{FIG.4 MASS.AVERAGED EXIT VELOCITY COMPONENTS}

and in the leakage vortex. In the other regions axial velocity exhibits a fairly uniform trend without any appreciable gradients in the radial and tangential directions. On the other hand, in the region outside the influence of viscous effects, the tangentail velocity exibits a steep gradient only in the radial direction. The radial velocity component shows a steep gradient at midspan in both the radial and tangential directions. Considerable local variations in radial velocity can be observed in the neighbourhood of the separated flow region at the hub, in the wake and at the casing where the leakage vortex is located.

Secondary flows, defined as the difference between the actual flow and a reference axisymmetric flow, are obtained considering the velocity components perpendicular to the relative reference flow direction. The reference direction is determined, at each radius, by the angle $\beta_{\text {ref }}$ in the blade to blade cylindrical surface and by the angle $\Psi_{\text {ref }}$ in the meridional plane. These angles are taken equal to the flow angle at midpitch in the inviscid flow region, and they are extrapolated linearly in the hub and tip regions where the flow departs from the inviscid conditions. The reference direction is used to determine the reference axisymmetric velocity distribution, which is obtained by integrating the radial equilibrium equation satisfying the continuity equation in the inviscid part.

The secondary flow vectors (Fig.6) are better represented if the flow is observed by introducing the rotation $\psi_{\text {rep }}$ the behaviour of the radial component at different radii and in the pitchwise direction can, therefore, be clearly seen. Near the wake radial flow can be observed towards the hub on the pressure side and towards the tip on the suction side of the wake. This is consistent with the clockwise pattem of the vortex that practically fills the entire free stream region and can be interpreted by considering the axial component of the vector equation $\nabla \times \vec{W}--2 \vec{\Omega}$ :

$$
\frac{\partial\left(W_{\theta} r\right)}{\partial r}+\frac{\partial W_{t}}{\partial \theta}=-2 \Omega r
$$


The agreement between the different terms of the equation and the experimental results in the central region can easily be verified. It is worth remembering that in those regions outside the influence of viscosity, where inlet flow can be assumed to be irrotational, deviation from the free vortex law corresponds to the radial velocity gradients in the circumferential direction.
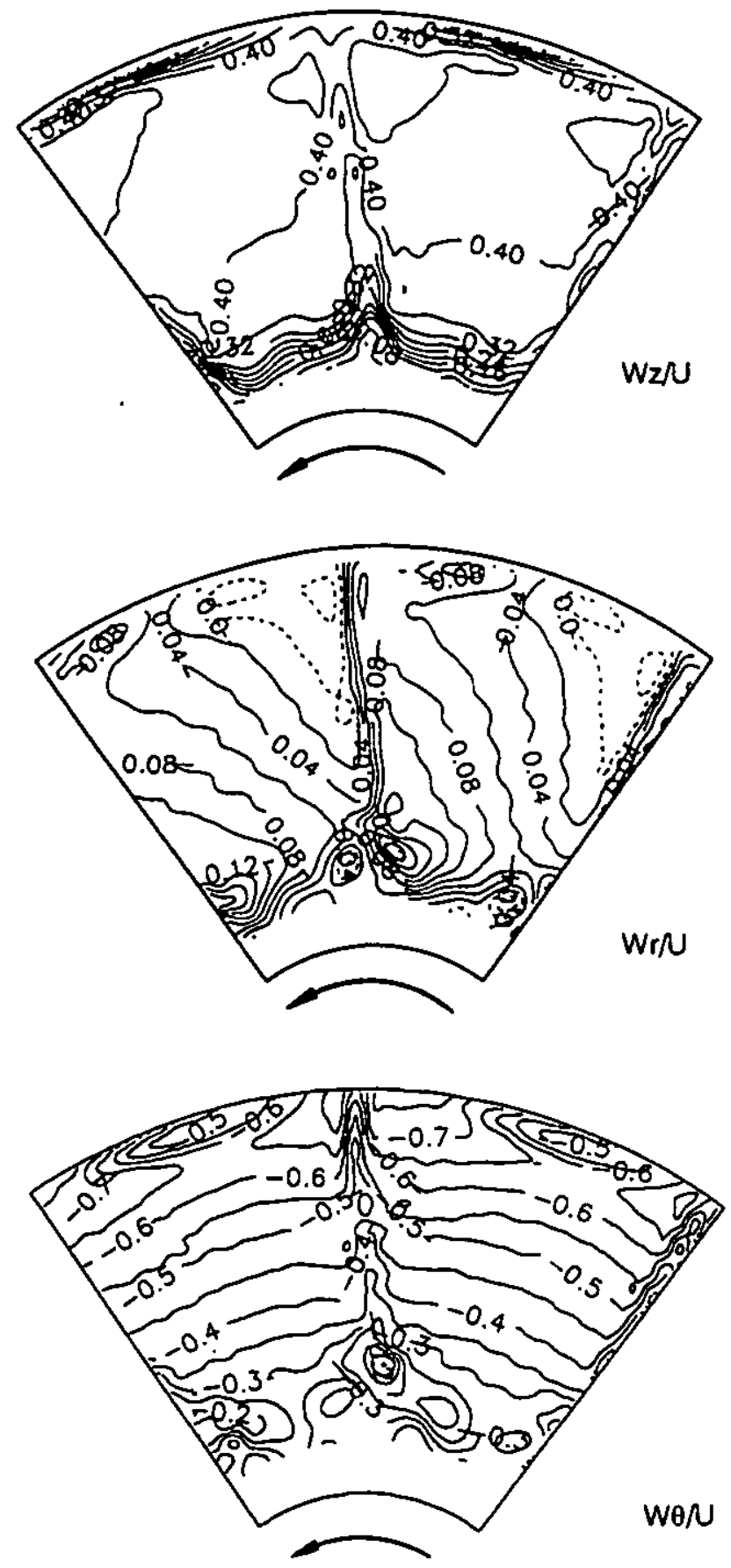

\section{FIG.5 CONTOUR PLOTS OF VELOCITY COMPONENTS AT THE ROTOR EXIT $\left(\gamma=62^{\circ} \Phi_{M}\right)$}
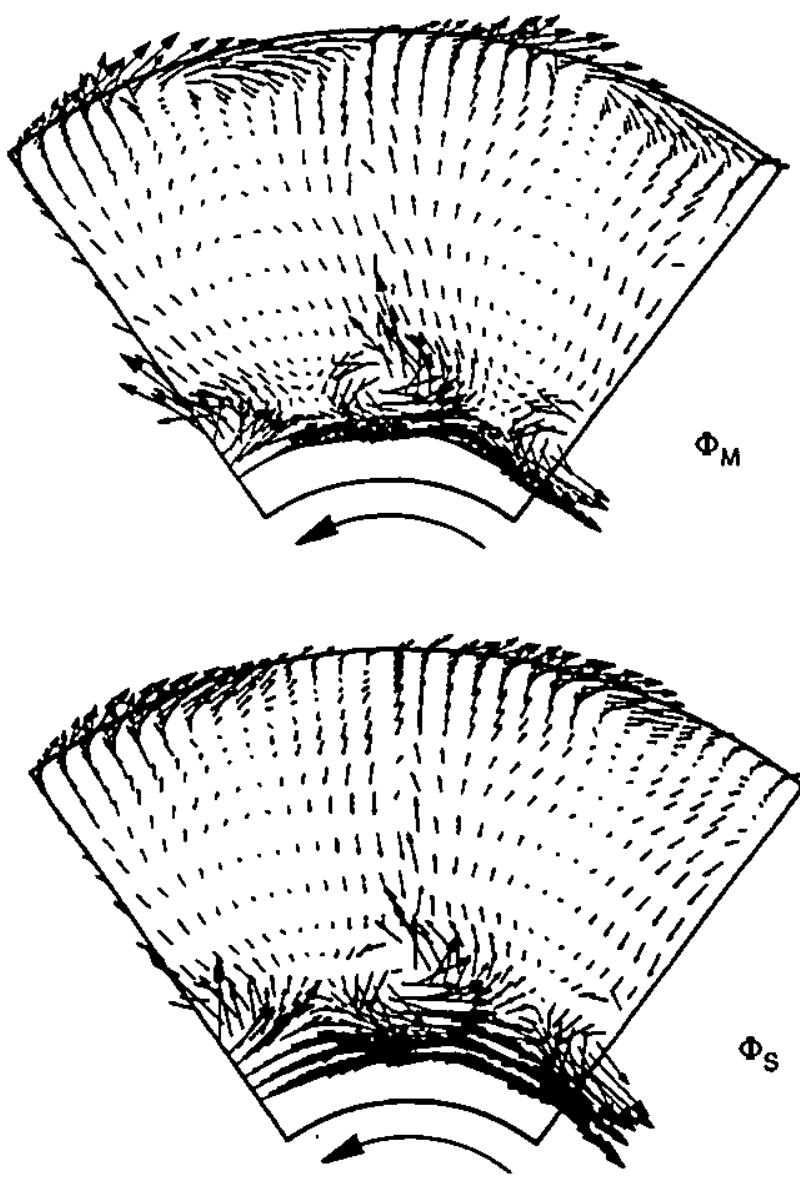

FIG.6 SECONDARY FLOW $\left(\gamma=62^{\circ} \Phi_{M}\right.$. $\left.\Phi_{S}\right)$

Regarding the flow field near the hub, with the exception of the separated region, the counter-clockwise trailing shed vortex can be clearly observed. For the high flow rate condition $\left(\Phi_{M}\right)$ the trailing vortex is located at $h / h o=0.25$ and is extended for $25 \%$ of the blade height and more than $50 \%$ of the pitch. It is also possible to recognize the clockwise passage vortex located between the neighbouring trailing vortex but characterized to a lesser extent.

When the blade loading is increased $\left(\Phi_{S}\right)$ the trailing vortex grows larger and stronger, it shifts upwards $h / h o=0.3$ and practically fill the entire blade pitch. The passage vortex disappears because of the stronger interaciion between the trailing vortices and the large vortex in the free stream flow.

Near the casing, the leakage vortex strongly affects the tlowfield; its centre is clearly evidenced by the vector plots in which the significant radial flows induced in the flow field (Stauter, 1992) can also be observed.

The presence of vortices within the flow can also be verified through the circumferential distributions of the absolute velocity components. As an example, Fig. 7 shows the velocity distributions for the regions near the casing ( $h / h 0=0.95)$ and near the hub ( $h / h o=0.2$ ) for the stagger angle $y=62^{\circ}$ and the lower blade loading. For $h / h o=0.95$, the counter-clockwise 
trailing shed vortex in the wake and the clockwise leakage vortex at midpitch, which correspond with a steeper gradient of the velocity components, can be clearly seen.
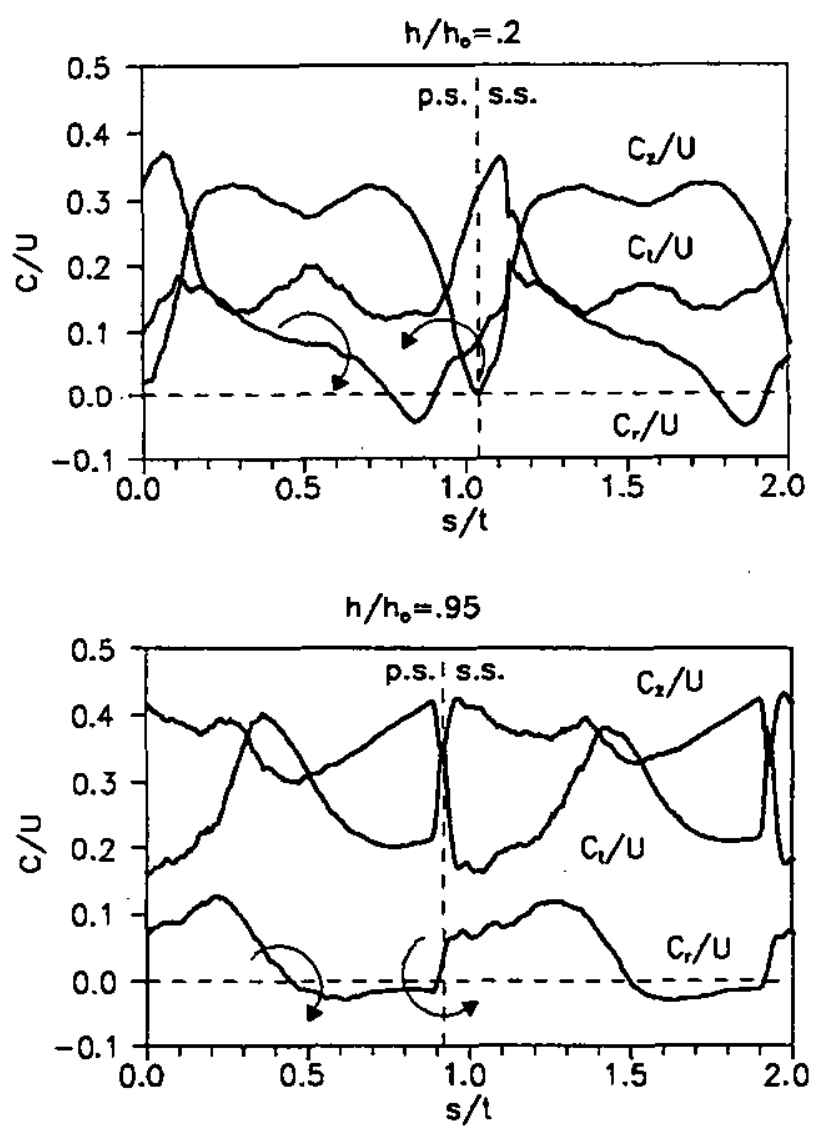

\section{FIG.7 CIRCUMFERENTIAL DISTRIBUTION OF ABSOLUTE VELOCITY COMPONENTS $\left(\mathrm{y}=62^{\circ} \Phi_{M}\right)$}

In the region of the hub ( $\mathrm{h} / \mathrm{ho}=0.2)$ a similar trend can be observed though the pitchwise gradients are gentler. Here both the trailing vortex and the clockwise passage vortex can be recognized. The lanter vortex can be identified from the pitchwise distortions existing in the distribution of the velocity components.

To examine the flow from the kinetic energy point of view let us consider the contour map of the kinetic energy defect

$$
\xi_{\mathrm{D}}=\frac{\mathrm{w}^{2}-\mathrm{w}_{\mathrm{ref}}{ }^{2}}{w_{\mathrm{ref}}{ }^{2}}
$$

where the relative velocities $W$ and $W_{\text {ref }}$ indicate actual and reference flows respectively.

The dashed lines denote the regions of high kinetic energy. In Fig. 8 the wake, the hub boundary layer as well as the leakage vortex and trailing vortex are clearly evidenced by the high energy defect.
Also worth noting is the region with low kinetic energy on the suction side of the wake associated with the accumulation of low energy fluid transported by the vortices.
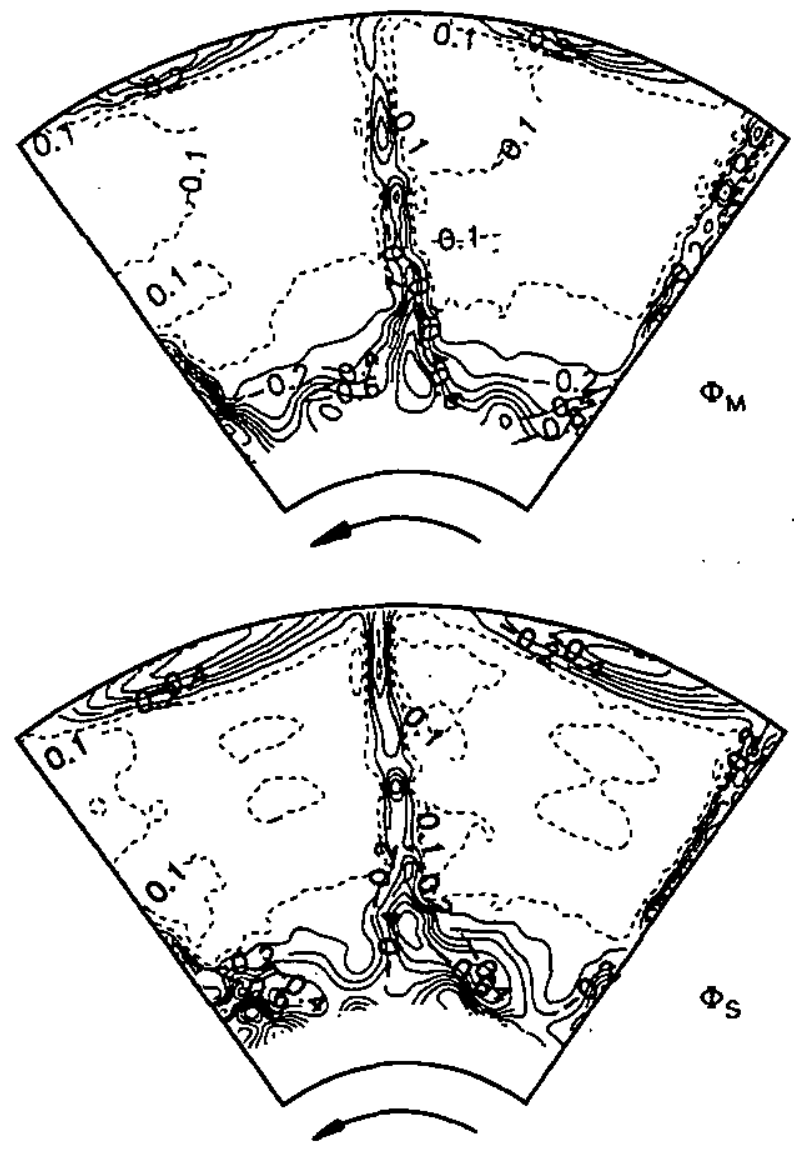

FIG.8 KINETIC ENERGY DEFECT $\left(y=62^{\circ} \Phi_{M}, \Phi_{S}\right)$

\section{Effects of changing blade loading and stagger engle}

The secondary flow pattern described above for operating condition close to the design one varies substantially when load and stagger angle are changed. With increasing loading (stagger angle $\gamma=62^{\circ}$ ) the regions affected by the trailing vortex and by the leakage vortex extend over a wide part of the pitch and at the same time the separatinn region at the hub slightly increases. The vortices keep the same structure but they exert a greater infiuence on the overall flow field.

The kinetic energy defect contour maps allow to estimate the extent of the regions affected by the vortices in question and their position. In particular at low blade loading a region with high kinetic energy defect on the suction side will increase in size at higher blade loading. This latter effect is due to the interaction between the enlarged trailing vortex and the separation zone.

Interesting results were obtained for the other stagger angle $\left(y=70^{\circ}\right)$ shown in Fig.9. Here too the effects of trailing vortices on the flow field can be clearly recognized and are accentuated at higher blade loading. At the $\Phi_{M}$ operating point the trailing 
vortices are less extended over the pitch than in the previous case and, therefore, the passage vortex gains more importance and it is placed at midpitch between the other two with the centre at about $40 \%$ of the blade height.

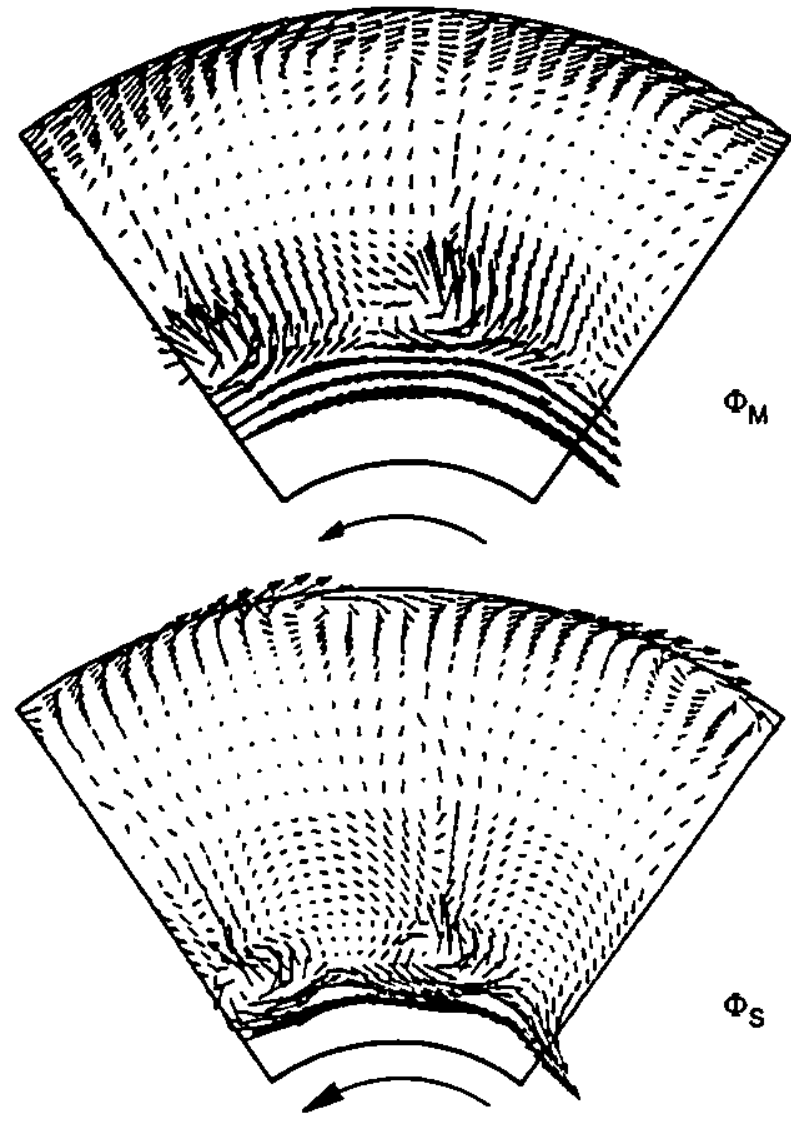

FIG.9 SECONDARY FLOW $\left(\gamma=70^{\circ} \Phi_{M} \cdot \Phi_{S}\right)$

The leakage vortex after rolling-up can be observed in this case in the region shifted towards the blade pressure side.

As the blade loading is increased, the flow structure undergoes an interesting modification which calls for more detailed analysis. The separation zone at the hub is more accentuated causing a radial displacement of the centre of the trailing edge vortices which are now located at $40 \%$ of blade height. Radial velocity differs substantially (Figs.10,11) and also the pitchwise gradients over the whole flow field. The presence of a wider separation zone at the hub is also responsible for the appreciable variation of the inlet pitcb angles. As for the smaller stagger angle tested, the passage vortex cannot be clearly defined at high blade loading.

For $y=70^{\circ}$, the secondary vorticity components ( $\left.\omega_{\text {acan }}-\omega_{\text {ref }}\right)$ are also reported, in Fig.12, through their projections along the average relative fow directions $(s, \tau, n)$.

The secondary vorticity is here defined, (Smith, 1955), as the difference between the absolute vorticity of the actual flow and the absolute vorticity of the axisymmetric reference flow.
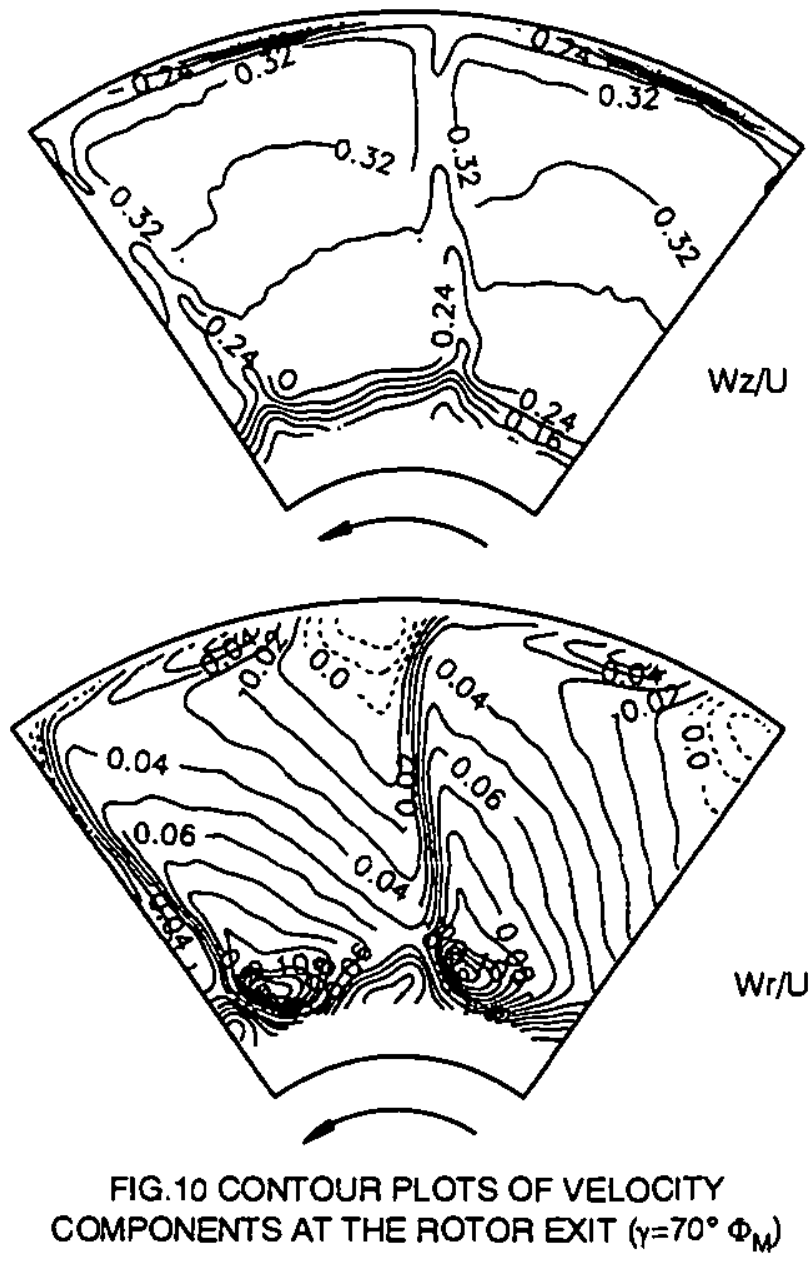

The vorticity components $\omega_{2}, \omega_{\theta}$ and $\omega_{r}$, were estimated by numerical differentiation then smoothed and projected along the $s, \tau, n$ directions of the reference flow (see appendix).

The dashed lines characterize the regions with negative vorticity values (counter-clockwise). As can be observed, the trailing voriex is essentially characterized by strong positive streamwise vorticity. In the casing region strong transverse vorticity exists and this can be attributed above all to the leakage vortex and its interaction with the flow beneath it. While the streamwise vorticity component is influenced by the leakage vortex rolling up, the transverse component affects more or less $20 \%$ of the blade span, yielding a more accurate measure of the interaction region of the tip secondary flows with the free stream region flow.

\section{Remarks}

Looking at the experimental results for both the mean flow and relative flows in the blade vane it is possible to draw some further considerations, comparing the flow angles and the blade angles. 

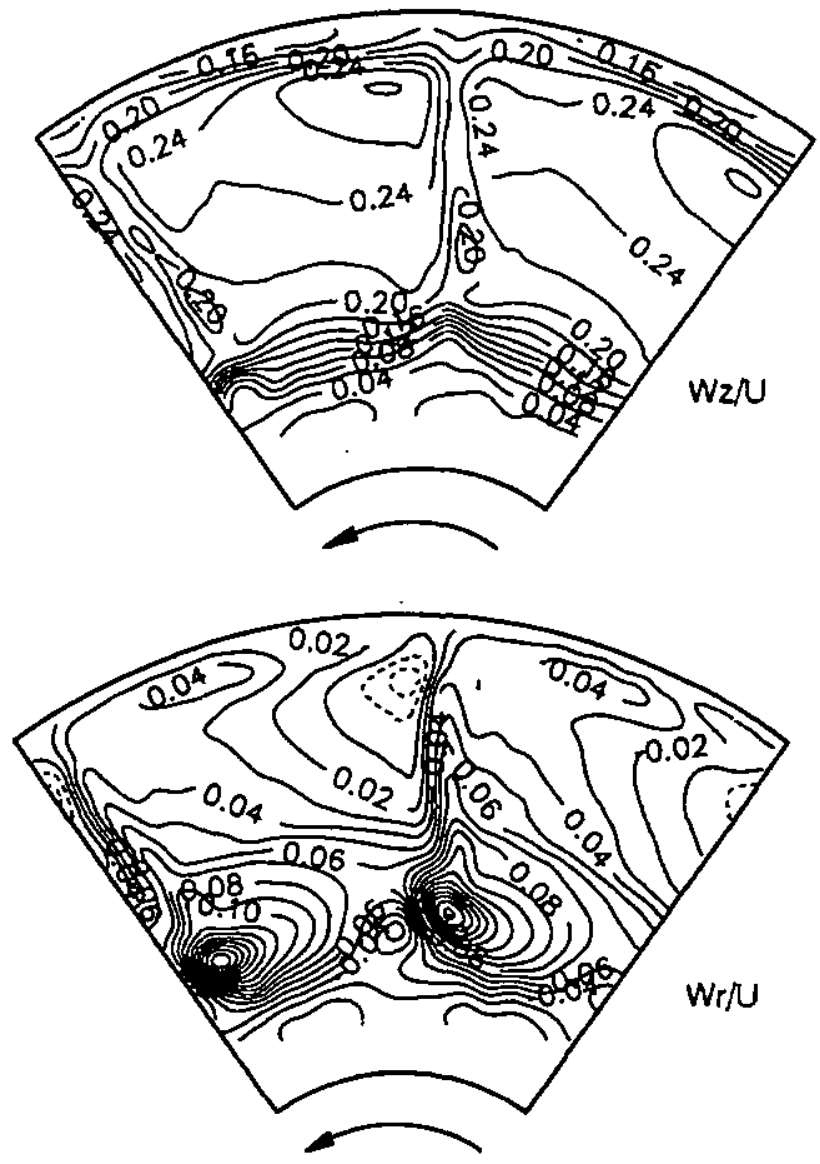

\section{FIG.11 CONTOUR PLOTS OF VELOCITY COMPONENTS AT THE ROTOR EXIT $\left(\gamma=70^{\circ} \Phi_{S}\right)$}

The axial flow fan, which was designed some decades ago placing greater enphasis on manufacturing requirements than on achieving the accomplishment flow radial equilibrium, has geometry and operating conditions that generate a large region of separated flow at the hub.

The extent of such a region, however, depends both on the aerodynamic load and the stagger angle and also influences the free stream flow region.

The mean relative flow angle distribution is strongly influenced by the three-dimensional effects produced, to a large extent, by the spanwise variation of the blade circulation. Comparing the relative flow angle with the blade angle, a strong distortion can be observed near the trailing vortex location. In Fig.13 for $\gamma=70^{\circ}$ and low flow rate $\Phi_{S}$ the angle of deviation $\left(\beta-\beta_{\text {blade }}\right)$ is almost constant between $b / h o=0.9$ and $h / h o=0.5$, decreases from $h / h o=0.5$ down to $h / h o=0.35$ and then increases. Similar behaviour, also observed in the other test conditions, suggests that the secondary flows affect the angle of deviation and the blade loading for an extended region of the blade height. Furthermore for this last test condition the pitch flow angle at the rotor inlet and exit, exhibits a different trend along the radius compared to the other flow conditions. This behaviour is significant because, when the stagger angle and

the load are increased, the structure of radial flow is seen to alter in the hub region and this phenomenon is also associated to the displacement of the trailing vortices.

This flow pattem is interesting because it can be related to the different behaviour of the flow in regard to the instability phenomena as can be verify throttling the fan.
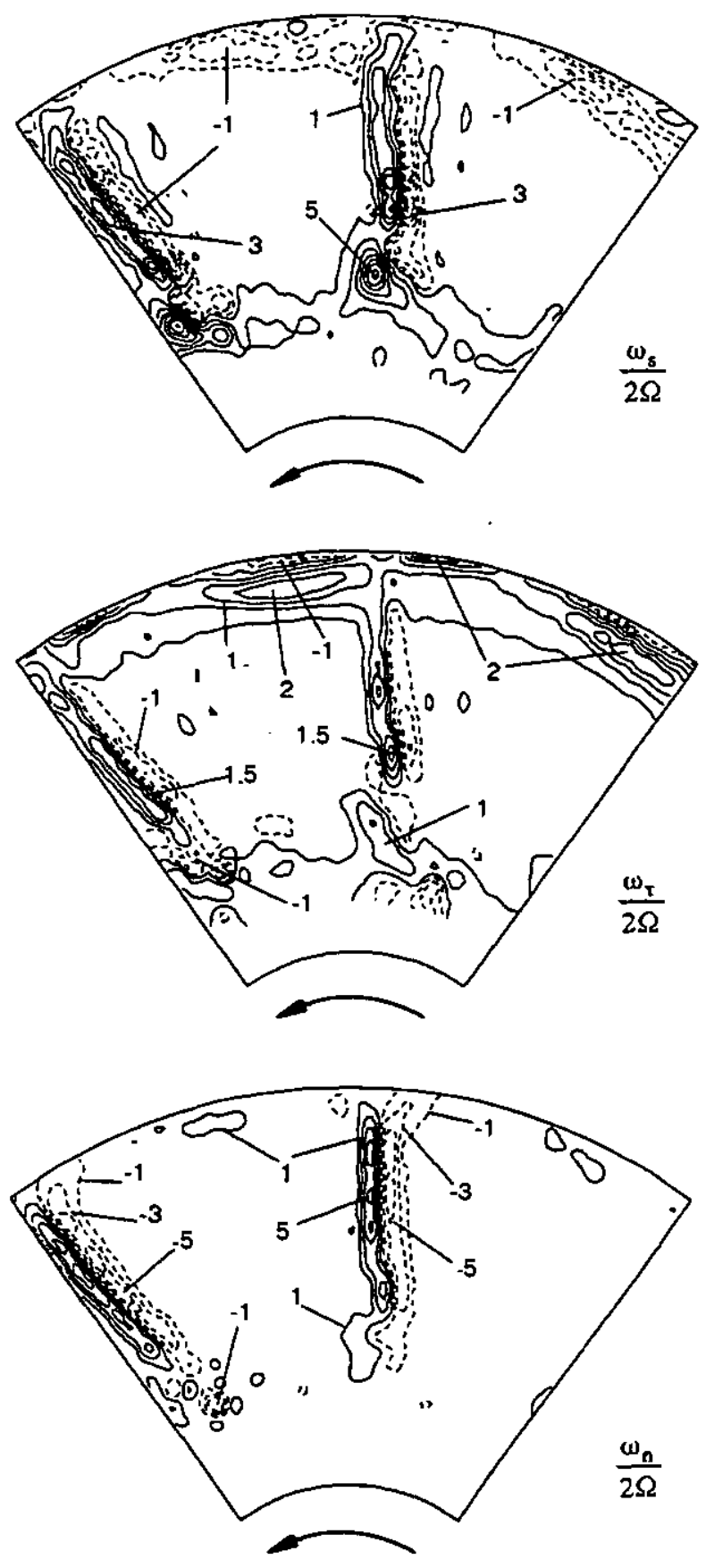

FIG.12 SECONDARY VORTICITY COMPONENTS $\left(\gamma=70^{\circ} \Phi_{S}\right)$ 


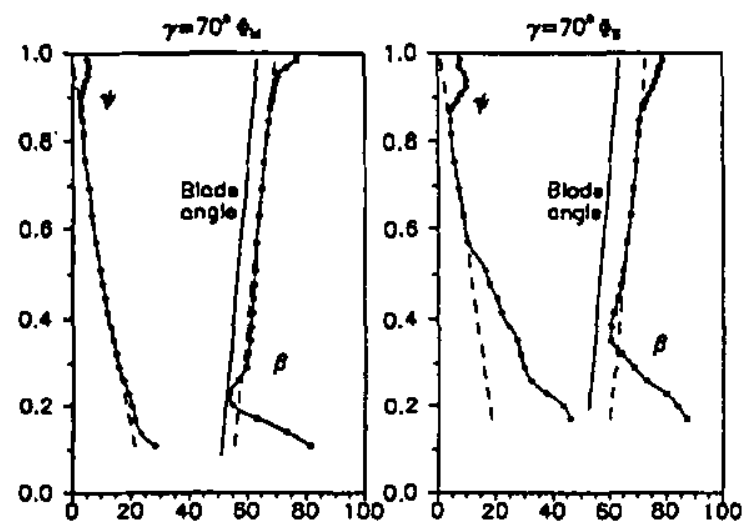

FIG.13 EXIT RELATIVE FLOW ANGLES

\section{CONCLUSIONS}

The relative flow in an industrial axial fan with 0.3 hub/tip ratio has been studied using the well-known phase-locked ensemble averaging technique with a slanted bot-wire anemometer.

Flow measurements were performed for two different stagger angles and blade loading conditions. The following conclusions may be drawn:

- mean flow over the blading exhibits a strongly threedimensional nature: an extensive separated flow region exists at the hub which increases at higher blade loading;

- secondary flows are present throughout the flow field and have a strong influence on flow pattem in the test conditions examined;

- in the midpassage region a vortical pattern, explained in terms of relative vorticity, can be clearly observed. This was consistent with the radial flow toward the hub on the pressure side and toward the tip on the suction side of the wake. Near the hub this vortical flow interferes with both the passage vortex, with which it partly merges, and the large trailing vortex;

- at higher loading the trailing vortices affect a broader region and because of the low hub/tip ratio may interact with each other $\left(\right.$ see $y=62^{\circ}$ );

- the secondary flows and in particular the trailiog vortex strongly affect the deviation angle distribution,

- the rolling up leakage vortex is well defined and can be clearly recognized in the secondary flow. Interaction of the tip leakage vortex and the trailing edge vortex with the free stream region flow determines a fairly broad region of strong vorticity that increases at higher stagger angle and blade loading;

- for stagger angle of $y=70^{\circ}$ and the higher blade loading, the separated flow region at the hub as well as the strong vorticity region at the tip are accentuated.

\section{ACKNOWLEDGEMENTS}

This research was conducted with the financial support of the Italian Ministry for University and Scientific Research and
National Research Council. We are also grateful to Woods for their assistance and technical collaboration.

\section{REFERENCES}

Adkins G.G. Jr., Smith L.H. Jr., 1982, "Spanwise Mixing in Axial-Flow Turbomachines", Trans. ASME, Journal of Engineering for Power, Vol.104, $\mathrm{N}^{\circ} 1$, pp. 97-110.

Chen, G.T., Greitzer, E.M., Tan, C.S., Marble, F.E., 1991, "Similarity Analysis of Compressor Tip Clearance Flow Structure", ASME Jounal of Turbomachinery Vol.113, $\mathrm{N}^{\circ} 2$, pp.260-271.

Dawes, W.N., 1987, "An Numerical Analysis of the ThreeDimensional Viscous Flow in a Transonic Compressor Rotor and Comparison with Experiments", ASME Journal of Turbomachinery, Vol.109, $\mathrm{N}^{\circ} 1, \mathrm{pp} .83-90$.

Dring, R.P., Joslin, H.D., Hardin, LW., 1982, "An investigation of Axial Flow Compressors 'Rotor Aerodynamics", Trans. ASME, Journal of Engineering. for Power, Vol.104, N¹, pp.84-96.

Gallimore, S.J., Cumpsty, N.A., 1986, "Spanwise Mixing in Multistage Axial Flow Compressor: Part I-Experimental Investigation", ASME Journal of Turbomachinery, Vol.108, $\mathrm{N}^{\circ} 1$ pp.2-9.

Goto, A., 1992, "Three-Dimensional Flow and Mixing in an Axial Flow Compressor with Different Rotor Tip Clearances", ASME Joumal of Turbomachinery, Vol.114, N.3, pp.675-685.

Ha, C., 1986, "A Numerical Modelling of Endwall and TipClearance Flow of an Isolated Compressor Rotor", ASME Journal of Engineering for Gas Turbines and Power, Vol.108, $\mathrm{N}^{\circ} 1$, pp.15-21.

Hunter, I.H., Cumpsty, N.A., 1982, "Casing Wall BoundaryLayer Development Trough an Isolated Compressor Rotor", ASME, Journal of Engineering for Power, Vol.104 $\mathrm{N}^{\circ} \mathrm{pp} .805$ 818.

Inoue M., Kuroumaru M., 1984, "Three-Dimensiona! Structure and Decay of Vortices Behind an Axial Flow Rotating Blade Row" Trans. ASME, Journal of Engineering for Gas Tutbines and Power, Vol.106, N³, pp.561-569.

Inoue M., Kuroumaru M., 1989, "Structure of Tip Clearance Flow in a Isolated Axial Compressor Rotor "Trans. ASME, Joumal of Tutbomachinery, Vol.111, N³, pp.250-256.

Inoue M., Kuroumaru M., Fukuhara M., 1986, "Behaviour of Tip Leakage Flow Behind an Axial Compressor Rotor " Trans. ASME, Journal of Engineering for Gas Turbines and Power , Vol.108, $\mathrm{N}^{\circ} 1$, pp.7-13.

Lakshminarayana , B., Pouagare, M., Davino, R., 1982, "Three-Dimensional Flow Field in the Tip Region of a Compressor Rotor - Part Il Turbulence Properties" ASME, Journal of Engineering for Power, Vol.104 N4, pp.772-781.

Leylek, J.H., Jr., Wisler, D.C., 1991, "Mixing in Axial-Flow Compressors Conclusion Drawn from 3-D Navier-Stokes Analysis and Experiments", ASME, Journal of Turbomachinery, Vol.113, pp.139-160. 
Lofdahl, L.,1986, "Hot Wire Techniques for the Determination of Reynolds Stress Tensor in Three-Dimensional Flows", Dantec Information $\mathrm{N}^{\circ} 3$.

Pandya, A, Lakshminarayana , B., 1983, "Investigation of the Tip Clearance Flow Inside and a the Exit of a Compressor Rotor Passage. Part I: Mean Velocity Field" ASME, Journal of Engineering for Power, Vol.105 $\mathrm{N}^{\circ} 1$, pp.1-12.

Perdichizzi, A., Ubaldi, M., Zunino, P., 1990, "A Hot Wire Measuring Technique for Mean and Reynolds Stress Components in Compressible Flow", Proc. 10th Symposium on Measuring Techniques for Transonic and Supersonic Flows in Cascades and Turbomachines, V.K.I., Brussel, Paper $\mathbf{N}^{\circ} 8$.

Puddu P., 1992, "Misure con sonde a filo caldo a valle di rotori di ventilatori assiali", Giornata di studio su "Tecniche avanzate per lo studio fluidodinamico dei ventilatori", Cagliari.

Puddu P., 1993, "Misure di turbolenza in elementi fissi e rotanti di turbomacchine con l'impiego di sonde a filo caldo", MIS-MAC II Firenze.

Smith L.H., 1955, "Secondary Flow in Axial-Flow Turbomachinery", Trans. of the ASME, pp.1065-1076

Stauter R.C., 1992, "Measurement of the Three-Dimesional Tip Region Flowfield in an Axial Compressor", ASME Paper $\mathrm{N}^{\circ} 92-\mathrm{GT}-211$

Waltis R.A., 1983, "Axial Flow Fans \& Ducts", John Wiley . \& Sons.

Wiesler D.C., Bauer R.C., Okiishi T.H., 1987, "Secondary Flow, Turbulent Diffusion, and Mixing in Axial-FlowCompressors", ASME Journal of Turbomachinery, Vol.109, $\mathrm{N}^{\circ} 4$ pp. $455-482$.

\section{APPENDIX}

The absolute vorticity components are thus defined:

$$
\bar{\omega}=\nabla \times \bar{C}=\left\{\begin{array}{l}
\omega_{z}=\frac{1}{r} \frac{\partial}{\partial r}\left(\mathrm{rC}_{\theta}\right)-\frac{1}{\mathrm{r}} \frac{\partial \mathrm{C}_{\mathrm{T}}}{\partial \theta} \\
\omega_{\theta}=\frac{\partial \mathrm{C}_{\mathrm{r}}}{\partial \mathrm{z}}-\frac{\partial \mathrm{C}_{\mathrm{z}}}{\partial \mathrm{r}} \\
\omega_{\mathrm{r}}=\frac{1}{\mathrm{r}} \frac{\partial \mathrm{C}_{\mathrm{z}}}{\partial \theta}-\frac{\partial \mathrm{C}_{\theta}}{\partial \mathrm{z}}
\end{array}\right.
$$

They can be trasformed in the streamwise, transverse and normal direction by the following relation:

$$
\begin{aligned}
& \left\{\begin{array}{l}
\omega_{\mathrm{s}} \\
\omega_{\mathrm{r}} \\
\omega_{\mathrm{n}}
\end{array}\right\}-[\mathrm{T}]\left\{\begin{array}{l}
\omega_{\mathrm{z}} \\
\omega_{\theta} \\
\omega_{\mathrm{r}}
\end{array}\right\} \text { where }[\mathrm{T}] \text { is the conversion matrix } \\
& \therefore \\
& \quad \mathrm{T}=\left[\begin{array}{ccc}
\cos \beta_{\mathrm{ref}} \cos \psi_{\mathrm{ref}} & -\sin \beta_{\mathrm{ref}} & \cos \beta_{\mathrm{ref}} \operatorname{sim} \psi_{\mathrm{ref}} \\
\sin \beta_{\mathrm{ref}} \cos \psi_{\mathrm{ref}} & \cos \beta_{\mathrm{ref}} & \sin \beta_{\mathrm{ref}} \operatorname{sim} \psi_{\mathrm{ref}} \\
-\operatorname{sim} \psi_{\mathrm{ref}} & 0 & \cos \beta_{\mathrm{ref}}
\end{array}\right]
\end{aligned}
$$

